\title{
Papers
}

\section{Ingest, manage and distribute}

\author{
Skiff Wager \\ has extensive experience in the news, print, music and cable markets for corporations like Xerox, Universal, Scripps Networks and \\ others. He was an integral part of the global roll-out of an EDS/Xerox engagement, held the Vice President of eCAT (e-Commerce and \\ Advanced Technology) responsibility at Universal Studios and was fortunate to be a part of his fourth launch of an asset management \\ initiative at EW Scripps (HGTV, Food Network, Do-it-Yourself-Network and Fine Living). Skiff has 26 years of IT experience and provides \\ a wide array of expertise in business and solutions architecture.
}

Keywords: ingest, manage, distribute, creation, measurement, asset, model, e-master

Abstract The author's previous paper "Digital asset management, media asset management and content management: From confusion to clarity" provided a simple to understand method of defining the differences between these asset management types. DAM was defined as the overall business strategy that provides direction to specific implementations of both media asset management and/or content management. Within these implementations comes the use of a model allowing a customer to focus their research specific areas. The ingest, manage and distribute model is very effective in support of this activity and is discussed in detail in this paper.

\section{INTRODUCTION}

In the author's previous paper ${ }^{1}$ digital asset management (DAM) was shown to be the key business strategy providing direction for the specific implementations of both media asset management and/or content management (see Figure 1). The DAM business strategy helps in defining the targeted goals of the company from an asset perspective and provides the umbrella guidance to specific initiatives. This definition helps drive the thought process in how best to approach the task of executing your due diligence in the specific areas of asset management.

Once your DAM business strategy has been well defined through a workshop-type process to uncover the specifics of the business strategy and the buy-in and concurrence of the business owners, you will have the ability to narrow your focus and determine your next steps. These are likely to be evaluating your priorities within the business strategy. The top priority should directly align with a specific department or need and will allow you to begin the process of executing the due diligence required to solve the business need in that area. Having the ability to narrow your focus will reduce the overall effort in the research of appropriate solutions and services. With the cross-over and blending of supplier strategies, their software and solutions, the research into viable solutions can get very confusing. The key to keeping this effort in check can start with the question "where does the need and opportunity fall" within the key building blocks of a high-level architecture. Figure 2 shows an industry standard positioning workflow.

\section{CREATE, INGEST, MANAGE, DISTRIBUTE, MEASURE}

Figure 2 provides the framework to place an event or activity in one of the specific areas. In your workflow you will find that each event will occur in one of these areas and at times will cross from one area to another. For example, in a particular scenario you may say to yourself this workflow is pertinent to a basic day-to-day process. I create a Word document, ingest it into my workstation, I manage it in Explorer, I email it to my colleague and I measure it by checking to see if they received it (see Figure 3). This seems pretty straightforward. However, if you 


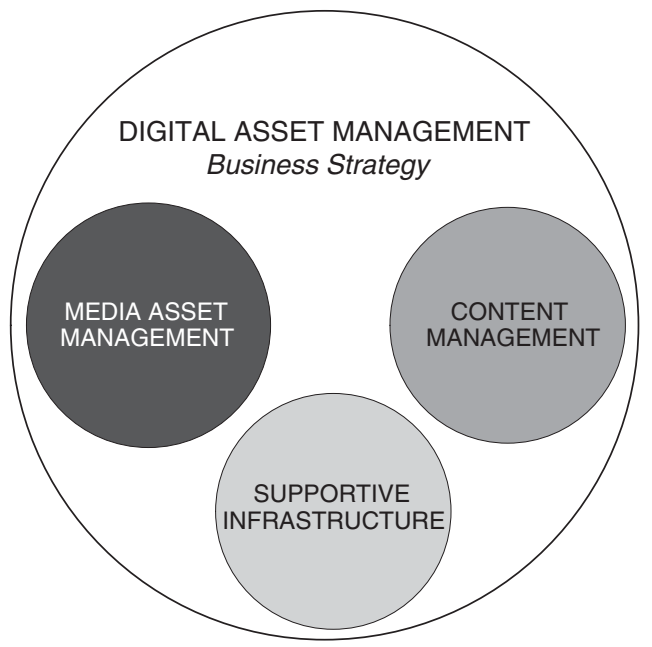

Figure 1: DAM business strategy model

back up from the trees and look at the forestsay an entire division - you can begin to imagine volumes and volumes of data needing the same movement and management.

\section{THE FOREST FROM THE TREES}

On a large scale with a myriad of data types, the ability to provide checks and balances along the high-level workflow can become quite a task. You want to ensure that the data that is being moved and managed in each step is accurate, intact and is where it is supposed to be. The old saying "garbage in, garbage out" still very much rings true. Your asset management archive, whether it is local or part of a much larger divisional archive, can get corrupted very quickly with the currently available methods to move data. One of the key elements to any asset management archive is organization. If your data are not organized correctly and continuously, system users will get undesirable results when requesting content. It is therefore very important to provide checks and balances from one key area to another, as there are different uses of both the content and the metadata, as well as different users throughout the workflow.

Figure 2 provides the building blocks for positioning large data movement. Within each one of these key areas there is much activity with specific validation points internal to each area. But let's first look specifically at how asset management applies to this model.

\section{DIGITAL ASSET MANAGEMENT, THE CATCH-ALL}

In the early days of asset management, it was thought that all assets within a company should be included in the asset management initiative. This meant everything from video and audio, to say royalty information, graphics, human resource data, flat text, etc. The idea was that as the term "asset" was being defined, individuals that had project ownership began to think, "well, this is an asset - it should be part of this new initiative." Everyone wanted to get in on

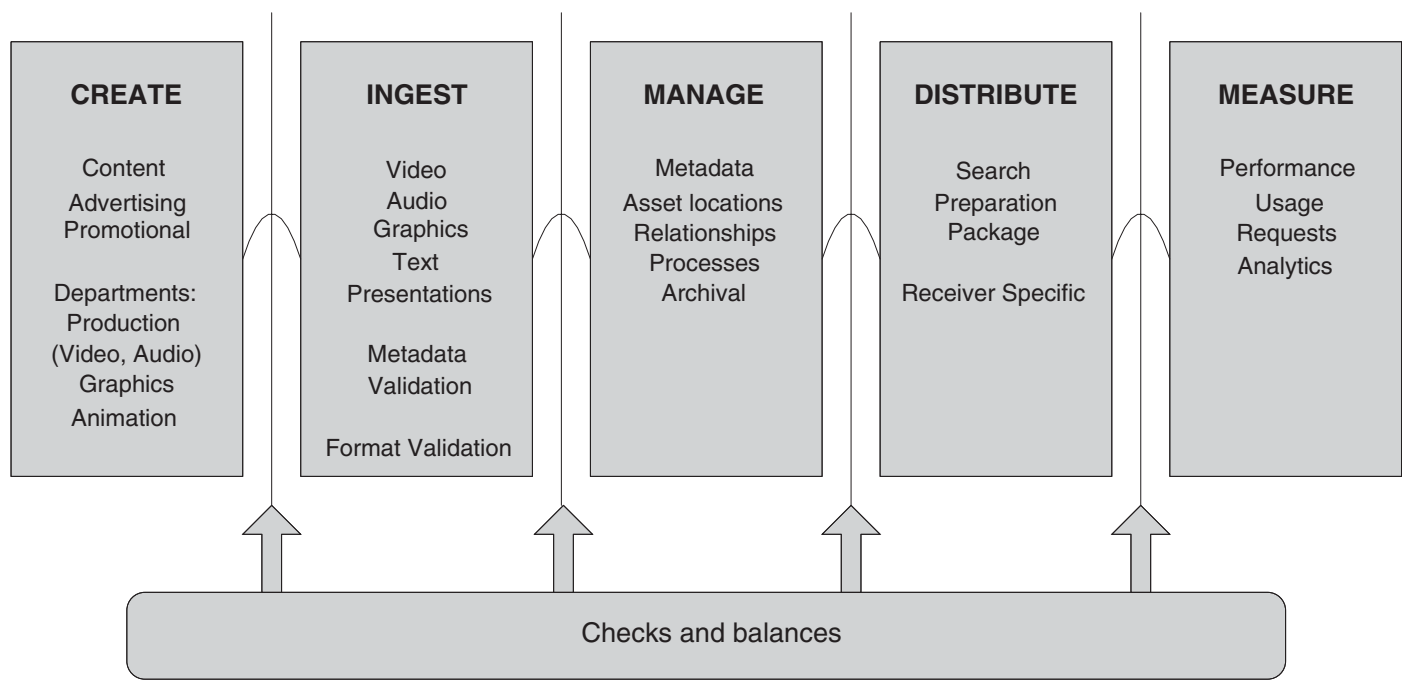

Figure 2: $\mathrm{QA} / \mathrm{OC}$ check points to create, ingest, manage, distribute and measure model 
the game. The concept being that the underpinning infrastructure, storage systems, networks, servers, software operating systems, etc, could be capitalized across the division for all departments, and the individual software tools (eg a scheduling application) could be expensed at the departmental level.

What this actually did, however, was drive up the costs for single line items so high that the financial community was taken aback and put the brakes on.

Consider storage, for example. Even if scrubbing through a year-end financial statement would show that totalling each line item related to storage (eg PC hard drives, server hard drives, SAN arrays with disk drives, etc) would add up to an overall single line item for storage, none of the financial bodies looked at costs in this way. Items were departmentalized. Thus, when the asset management initiatives initially began to bring the disparate silos together and roll overall costs to a single initiative, the numbers were astounding. Over the course of a couple of years DAM began to get a bad name. This was due to DAM being a technology-driven initiative and not a business one. As we have come full circle-digital asset management has matured and is now understood to be a business strategy not an application. If approached this way then senior executives will begin to understand that DAM as a business strategy makes sense and individual initiatives that fall under this strategy can be launched over time. In order to show how these initiatives financially share, underpinning infrastructures becomes the task of the initiative owner. This is where the create, ingest, manage, distribute and measure building blocks began and how they have morphed over time into the ability to show clearly where expense and capital outlays fall, and how you can show the overall growth of the DAM business strategy in a staged approach.

Having stated this, it was mentioned that the underlying infrastructure would support all of the departmental applications and that a company could achieve real cost efficiencies by negotiating contracts (eg for storage, servers, networking tools, etc) with their suppliers. This rings true to a point. So to re-address the model in Figure 2, let's take a look at where an enterprise-level asset management application falls within this model and how to align these applications specifically within the workflow.

\section{INGEST, MANAGE AND DISTRIBUTE-THE FOCUS OF ENTERPRISE ASSET MANAGEMENT}

When we look at the model in Figure 2, we want to focus on the "ingest, manage and distribute" portions, but need to explain how and why we peel off the creation and measure building blocks. This is directly related to content workflow and what is to be achieved in each area of the building block model. In very general terms you expect an asset management application to provide organization of data, to provide this data for use by multiple entities and secure and report any event that occurs.

However, the ability to ingest particular types of data and the manner in which these data need to be retrieved and specifically used begins to draw "glass walls" within the model.

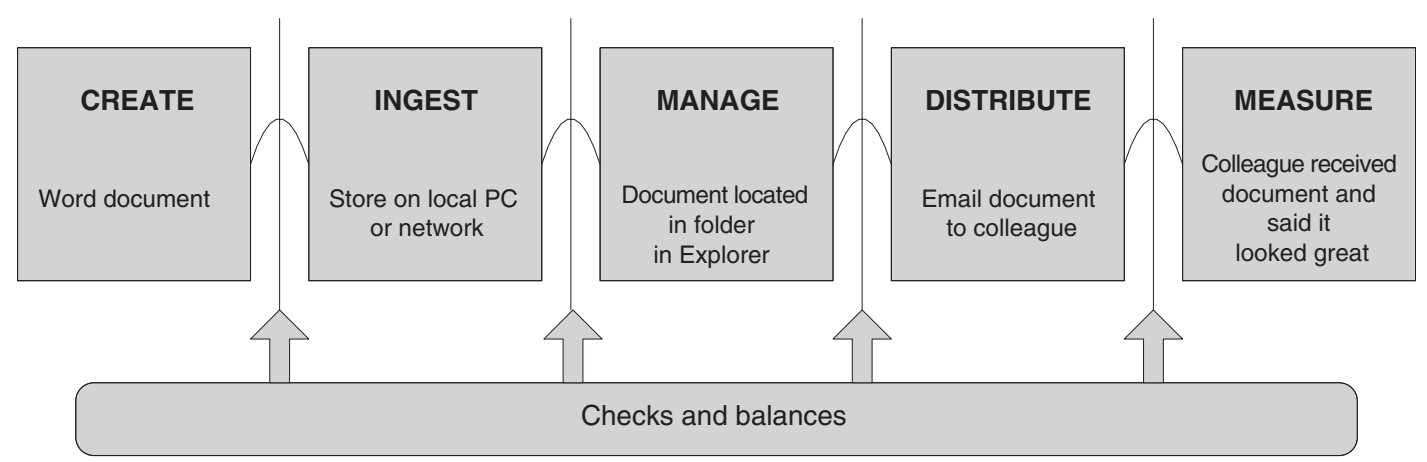

Figure 3: Sample use of model 


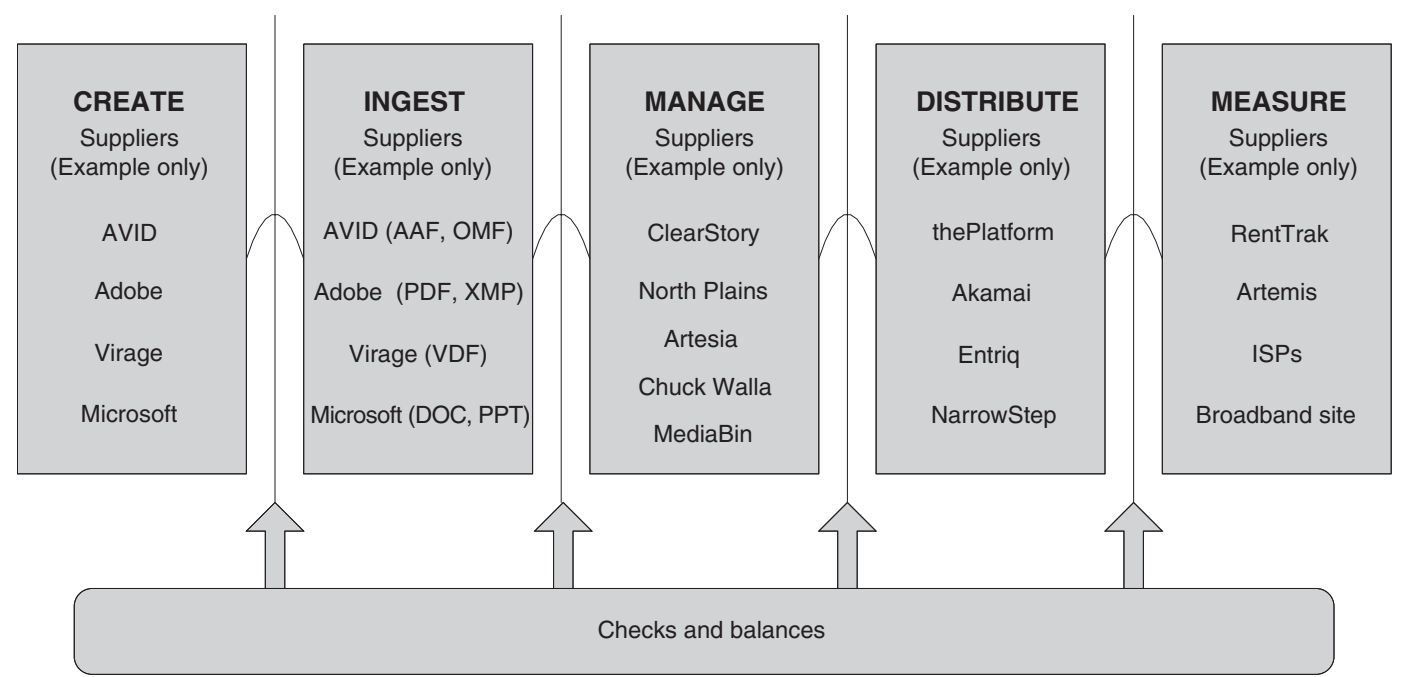

Figure 4: Sample suppliers within model

Enterprise asset management applications such as Clearstory, Artesia and North Plains, to name a few, are applications that manage "master" level content. This is not to say that versions of an electronic master or "e-master" are not represented within the applications listed above but the key is to provide this e-master level content at a minimum so that downstream use of content, (compressed video, PDF formatted files, etc) can be achieved. This brings up the context of ingest, manage, distribute, in the DAM business strategy. As initiatives are launched, one of the core applications will be an asset management application like one mentioned above and can certainly be the most important. This application will feed the majority of users over time, compared with applications that may reside, for example, in the production department. Therefore, take the approach that the ingest, manage and distribute blocks in Figure 4 relate specifically to these enterprise-level applications.

The create block in Figure 4 represents the departments such as traditional production and graphics departments, and others, and due to the iterations of content, it may be best to segment this from the core asset management systems that will be used by the majority of individuals. In the production-type departments, files and data are constantly being rendered or edited or altered in some fashion. Uncompressed files can be enormous and the number of users who access this data is relatively small in comparison with the overall community. Therefore, segment this semi-private area in its own environment allowing the constant change of files or versions to settle down into a final e-master type format prior to being ingested into the semi-public area which will have the larger volume of downstream users.

\section{INGEST}

Applications like Clearstory, Artesia, North Plains, Chuck Walla, MediaBin, etc are "management" systems. They rely on suppliers who provide tools to prepare content to be managed. They partner with and at times even acquire suppliers to provide the linkage between the tools to allow the ingestion of metadata into these management systems. This is important as one of the primary pieces of content is the "master" file.

It is important to start with the master file as there is a hierarchy within the applications which drives "parent/child"-type relationships. Starting with the "parent" master file, metadata and "child" assets are added to the system over time. A child asset, for example, may be a compressed Windows Media file which originated from an MPEG-2 file. The key here is the metadata.

In order to ingest content in an organized, efficient and error-reduced manner the asset management companies have partnered with 
suppliers who provide creation tools that prepare content for ingestion. This is the first "check and balance" in the compressed model indicated above. For example, suppliers such as Virage have developed encoders that take video masters (eg a CCR601 uncompressed file, $900 \mathrm{MB}$ for every 30 seconds of video), and encode this to an MPEG-2 file of say 12-MB bit-rate while also producing a Video Definition File (VDF). This VDF can automatically be read or ingested into the asset management application which much of the metadata about the video file.

Additionally, you may also concurrently encode a proxy, eg a Flash file, of the now MPEG2 emaster file which within the asset management application becomes a "child" to the "parent." By reducing human intervention and possible key-stroke errors you have mitigated the risk of putting "garbage" into your master archive. When architecting your asset management application, it is always the best practice to try and select tools that capture as much metadata at creation time about the incoming file as possible.

Another example in the ingest process is dealing with text-related files. Adobe Systems has come a very long way regarding metadata and has recently provided an extension to its metadata model to accomplish and support new processes. Some of the asset management applications have partnered with Adobe to provide linkage between their applications. Just as with Virage, Adobe has standardized on a metadata model for describing graphical and textual data. For example, the Adobe PDF file header holds specific metadata about the file and can be ingested directly to an asset management application and loaded specifically for the parent asset. The parent asset in this example is a Word document. This again mitigates the risk in misidentifying an asset, preventing negative consequences such as un-locatable assets, poor search results and rising creation costs. Adobe's recent progress has been supportive of the cohesiveness of the ingest, manage and distribute compressed model. Utilizing their new

eXtensible Metadata Platform (XMP)

framework has allowed metadata to travel with the asset, again greatly reducing the risk of the master content element being separated from the metadata describing the element.

Remember the asset management systems only hold the metadata about an asset, while the physical asset is stored in a separate storage location. Without going into too much detail about XMP, what has provided some real value is the "eXtensible metadata" which can now contain entries such as scheduling information, (what, where and when), and distribution data such as location of receiver, etc. This can greatly reduce ingest errors and in a sense is "prepping" content for packaging and distribution. Hold that thought as we will discuss the "distribution" particulars in a moment.

\section{MANAGE}

The core to the asset management applications is the management, organization and security of assets. These application suppliers partner with companies that provide tools for both the ingest as well as the distribution side of the model. These applications are very intelligent and can be structured to provide complex search models to support an array of customer types and needs. There are enterprise-level asset management applications and also departmental ones. For example, we mentioned that suppliers such as Clearstory, Artesia, North Plains and MediaBin are enterprise-level applications. Departmental asset management applications as an example are Avid's Unity for Video Production, Convera's Audio and Video Manager and Adobe's Media Server for Graphics. Each of these applications provides the management of metadata and physical assets. Due to the nature of content workflow it is prudent to segment the applications appropriately based upon customer usage.

Using the production department as an example, we previously mentioned that due to content workflow and customer usage, uncompressed content is constantly being revised and rendered until a final master is achieved. Therefore having a departmental management system such as Avid's Unity can manage that department's data only for the time period during editing. Once a final e-master is achieved, this physical asset and its metadata can be ingested into the enterprise-level asset management application. The Avid application can share the underlying divisional storage systems to keep costs down while expensing the software at the departmental level. The actual management of the data, however, will move from the departmental level to the enterprise 
level. This will allow the larger user community to access the enterprise-level application requesting searches for e-master parent assets such as an MPEG-2 file as well as related child assets such as Windows Media or Flash file formats. The smaller community, the production department, can have management tools specifically for that area and provide security designed for their use.

Thus, as we have defined the ingest side of the model, it pertains specifically to the accessing of content from one department to be "ingested" into the larger management area of the model to provide access to the wider community. The enterprise-level "management" application suppliers work closely with the ingest application suppliers to support the automated methods of ingesting content and metadata, reducing human error, mitigating risk of corrupting data and provide access to a wider array of content types for use by the larger downstream community.

\section{DISTRIBUTE}

In the compressed ingest, manage and distribute model we discussed the ingest companies and tool sets and departmental and enterprise management applications. The distribution applications and services which can deliver the content to specific receivers can extend these management applications. Briefly, a "receiver" can be any location that receives and utilizes downstream content from one entity to another. For example, a content provider may distribute content to a cable operator for on-air use, a broadband provider for web usage and articles for a printed magazine. These are all examples of receivers.

The management applications can provide the ability to search the systems to locate assets and allow the users to make decisions of what content could possibly be used where. The key is the metadata to assist the user in making these decisions. Without comprehensive metadata the user will not know their capabilities or limitations to distribute the content. Some of the enterprise-level management applications have the ability to package the search and requested content for distribution, but do not execute the actual distribution itself. This means that these applications do not actually move or transport the content to the desired location(s).
Additionally, comprehensive metadata can be applied to the content as it moves from the management system to the distribution system to provide value to the content and its receivers. This is actually true across the workflow.

Metadata are added and applied to the content as it moves from creation to management to distribution.

There are, for example, distribution suppliers such as thePlatform and Akamai that take the selected content from the management systems and apply metadata that is specific to the receiver and physically transport this content and metadata to the desired location. ThePlatform, for example, can apply metadata such as usage plans, licensing and/or receiver-specific information that will provide value to the content once delivered. These metadata are not applied at the time of ingestion as this information may not be known. In addition, these metadata are not entered into the management system as you may make downstream decisions up-to-the minute and apply this information in the distribution application or service. This information is time sensitive during the distribution activities and is measured upon usage. The measurement data or "take data" can be re-ingested into the management application but may be better used within an analytics or research database.

In summary we have explained the "create, ingest, manage, distribute and measure" (CIMDM) building block model and have applied various applications and services within these areas. The focus was to improve understanding, specifically the nuances of how this model applies to an enterprise asset management application. The asset management applications are not the catch-all of applications but are a core component to an overall DAM business strategy. The eventual architecture that is decided upon will incorporate numerous applications. Ones that assist in:

- the creation of content and the ability to store it and manage it locally within the department;

- the ingestion of this content at an e-master file type level and the automated loading of metadata mitigating risks of human error;

- the management of this mastered content along with child assets for downstream use for the larger 
community and assisting in decision making for distribution;

- the distribution of content with value-added metadata specific to the receiver and time sensitive information;

- the measurement of the distributed content as take data for use with analytic systems.

In your overall DAM business strategy you can apply a comprehensive return on investment analysis that can provide a wide view of how long-term, staged initiatives can be cost-effective and measured over time. You can link these systems (individually funded initiatives) together under your DAM business strategy as representing multiple initiatives and development efforts. From this strategy you can share the underlying infrastructure, capitalize the core components and expense the departmental tools which specifically apply to these departments. There are tools to help in this effort which can clearly define the multi-year investment and allow for initiative owners to measure the progress over time and make adjustments as necessary. In our next paper you can read the specifics on digital asset management investments: how to achieve success.

\section{Reference}

1 Wager, S. (2005) "Digital asset management, media asset management and content management: From confusion to clarity," Journal of Digital Asset Management, 1(1), 40-45. 